Article

\title{
Deep Belief Network for Spectral-Spatial Classification of Hyperspectral Remote Sensor Data
}

\author{
Chenming $\mathrm{Li}^{1}$, Yongchang Wang ${ }^{1}$, Xiaoke Zhang ${ }^{2}$, Hongmin Gao ${ }^{1, *} \mathbb{C}$, Yao Yang ${ }^{1}$ and \\ Jiawei Wang ${ }^{1}$ \\ 1 College of Computer and Information, Hohai University, Nanjing 211100, China; lcm@hhu.edu.cn (C.L.); \\ wangyongchang@hhu.edu.cn (Y.W.); rcyyang@hhu.edu.cn (Y.Y.); 142006020223@hhu.edu.cn (J.W.) \\ 2 School of Public Administration, Hohai University, Nanjing 211100, China; xkzhang@hhu.edu.cn \\ * Correspondence: gaohongmin@hhu.edu.cn; Tel.: +86-25-58099136
}

Received: 30 November 2018; Accepted: 3 January 2019; Published: 8 January 2019

\begin{abstract}
With the development of high-resolution optical sensors, the classification of ground objects combined with multivariate optical sensors is a hot topic at present. Deep learning methods, such as convolutional neural networks, are applied to feature extraction and classification. In this work, a novel deep belief network (DBN) hyperspectral image classification method based on multivariate optical sensors and stacked by restricted Boltzmann machines is proposed. We introduced the DBN framework to classify spatial hyperspectral sensor data on the basis of DBN. Then, the improved method (combination of spectral and spatial information) was verified. After unsupervised pretraining and supervised fine-tuning, the DBN model could successfully learn features. Additionally, we added a logistic regression layer that could classify the hyperspectral images. Moreover, the proposed training method, which fuses spectral and spatial information, was tested over the Indian Pines and Pavia University datasets. The advantages of this method over traditional methods are as follows: (1) the network has deep structure and the ability of feature extraction is stronger than traditional classifiers; (2) experimental results indicate that our method outperforms traditional classification and other deep learning approaches.
\end{abstract}

Keywords: hyperspectral image; deep learning; feature extraction; classification; remote sensors; multi-sensor fusion

\section{Introduction}

With the development of high-resolution optical sensors, hyperspectral remote sensing images are achieved, which consist of hundreds of different spectral bands of the same remote sensing scene. Hyperspectral remote images are essential tools for tasks such as target detection and classification because of these images' advantage in describing ground truth information. Their applications vary and include agriculture, military, geology, and environmental science. Different land covers have various spectral curves due to the complexity of the composition of the earth's surface. Hyperspectral images are rich in spectral information. Each pixel can produce a high-resolution curve. Traditional multispectral remote images use only a few bands to represent a complete spectral curve. However, dealing with hundreds of bands is also challenging [1]. Due to the large amount of hyperspectral remote sensing image data, the classification speed is slow. At the same time, the high spectral dimension of hyperspectral remote sensing images leads to the appearance of Hughes phenomenon.

Hyperspectral remote sensing images are collected by high-resolution optical sensors; the datasets used in this experiment were obtained by an airborne visible/infrared imaging spectrometer (AVIRIS) sensor and reflective optics system imaging spectrometer (ROSIS) sensor, respectively. 
Classifying hyperspectral images is a common technique in discovering information in hyperspectral sensor data. Prior to image classification, dimension reduction is necessary because hyperspectral images contain a large amount of information. Neural networks and support vector machines (SVMs) [2] are extensively used in hyperspectral classification because of their potential in handling high-dimension data. They can manage most of the classification but cannot provide enriched information. This problem renders these algorithms limited in several areas.

In recent years, research on deep learning received considerable attention due to breakthroughs in many fields. The use of deep learning in classifying hyperspectral images can result in high accuracy [3]. There are several common models in deep learning, such as the deep neural network model and recurrent neural network model. They have their own representative networks, convolution neural network and recursive neural network, respectively, referred to as CNN and RNN. CNN effectively reduces the problem of a large number of parameters. The convolution core is used as the intermediary. After image convolution, the original position relationship still exists, while the parameters of the hidden layer of the image input layer are reduced by geometric multiples. The basic operation units of $\mathrm{CNN}$ are convolution, pooling, full connection, and recognition. The recursive neural network can also be called a forward neural network. Sample processing time is independent. In RNN, the output of neurons can act on themselves at the next time. The recursive neural network can be regarded as a neural network that transmits in time. To improve the accuracy of classification, this work proposes the application of a deep belief network (DBN) model to hyperspectral images. DBN is an algorithm proposed by Hilton in 2006 [4]. It is based on a neural network and developed using the hierarchical learning method, which learns input data layer by layer in an unsupervised manner. Each layer is created using a restricted Boltzmann machine (RBM). The features learned are regarded as the input of the subsequent layer. Finally, a softmax classifier used in the last layer fine-tunes the parameters of the network in a supervised manner and labels each pixel and the result of the classification. In other words, DBNs are obtained by stacking RBMs on one another so that the input to one layer is given by the hidden units of the adjacent layer, as if they were data, and adding a last discriminative layer [5].

A Boltzmann machine is powerful in unsupervised learning and can locate information hidden among data. Thus, it is suitable for data mining. A Boltzmann machine is a fully connected network. This structure extends the training time, thereby restricting the application of the network. An RBM and its learning algorithm can address the problems of deep neural networks, such as classification, regression, image feature extraction, and collaborative filtering.

Many variants of the RBM were developed since the machine was created. The convolutional RBM created by Reference [6] can extract large-scale features and exhibit good performance. An effective method using the TLCNN-RBM (convolutional neural network mixed restricted Boltzmann machine based on transfer learning) model for a small sample of voiceprint recognition was provided by Reference [7]. In Reference [8], a Gaussian RBM was proposed to learn multiple layers of features from small images, whereas, in Reference [9], a conditional RBM learned to represent spatial transformations using factored high-order Boltzmann machines.

The main contribution of this work is the development of the stacks of an RBM, hereafter called the DBN model. We modified the standard RBM and its learning algorithm. The processes can be regarded as pretraining and fine-tuning, wherein data are trained in mini-batches to optimize the loss function of the validation dataset. The framework learns deep features that model different ground-truth classes in hyperspectral images. We experimentally demonstrate that this generative feature learning for a spatial classifier (SC) or joint spectral-SC (JSSC) becomes effective, using the learned features to exhibit state-of-the-art performance on hyperspectral image classification.

The rest of this paper is organized as follows: in Section 2, the main ideas and the structure of the DBN are discussed in detail, and the proposed hyperspectral sensor data classification method is presented in combination with spectral information and the spatial context. In Section 3, experimental results are elaborated. Finally, the study is summarized in Section 4. 


\section{Methods}

This section introduces the composition of RBM and the common DBN model, and then introduces the DBN classification model based on spatial information and joint spatial-spectral information.

\section{1. $R B M$}

An RBM is a random generative neural network composed of two layers. One layer comprises binary visible units, and the other comprises binary hidden units. An energy function was introduced to identify the state of the RBM, which was developed from the energy function of the Hopfield network in a nonlinear dynamic system. Therefore, the objective function of the system was transformed into an extreme value problem, and the RBM model could be easily analyzed [10].

An RBM is regarded as an Ising model; hence, its energy function is expressed as

$$
E(v, h ; \theta)=-\sum_{i j} W_{i j} v_{i} h_{j}-\sum_{i} b_{i} v_{i}-\sum_{j} a_{j} h_{j}
$$

where $\theta=(w, a, b)$ is the parameter of the RBM; $w_{i j}$ represents the value of the connection between the visible units $v$ and the hidden units $h$; and $b_{i}$ and $a_{j}$ are bias terms of the visible and hidden units, respectively.

The conditional distributions of the hidden units $h$ and the visible units $v$ are expressed as

$$
\begin{aligned}
& \mathrm{P}\left(h_{j}=1 \mid v\right)=\frac{1}{1+\exp \left(-\sum_{i} W_{i j} v_{i}-a_{j}\right)}, \\
& \mathrm{P}\left(v_{i}=1 \mid h\right)=\frac{1}{1+\exp \left(-\sum_{j} W_{i j} h_{j}-b_{i}\right)} .
\end{aligned}
$$

The target function of the RBM focuses on the solution of the distribution of $h$ and $v$ and renders them as equal as possible. Thus, we calculated the K-L (Kullback-Leibler) distance of their distribution and then reduced it.

In determining the expectation of the joint probability, obtaining the normalization factor $Z(\theta)$ is difficult, and the time complexity will be $O\left(2^{m+n}\right)$. Hence, Gibbs sampling was introduced to approximately reconstructed data. The learning of weights is expressed as

$$
\Delta w_{i j}=E_{\text {data }}\left(v_{i} h_{j}\right)-E_{\text {model }}\left(v_{i} h_{j}\right)
$$

The subtracted value was equal to the expectation of the energy function of the input data and could be obtained. This value was equal to the expectation of the energy function of the model, which was obtained from Gibbs sampling.

Training the RBM through Gibbs sampling is time consuming. We commonly use the contrastive divergence (CD-k) algorithm, where $\mathrm{k}$ is equal to 1 ; thus, the training time of the RBM network improves [10].

The average sum of the gradients was approximated using the samples obtained from the conditional distributions, and Gibbs sampling was performed only once.

\section{2. $D B N$}

A DBN is a probability generation model that is opposite the traditional discriminative model. This network is a deep learning model that is stacked by RBM and trained in a greedy manner. The output of the previous layer was used as the input of the subsequent layer. Finally, a DBN network was formed.

DBN hierarchical learning was inspired by the structure of the human brain. Each layer of the deep network can be regarded as a logistic regression (LR) model. 
The joint distribution function of $x$ and $h^{k}$ in Layer $l$ is

$$
p\left(x, h^{1}, h^{2}, \ldots, h^{l}\right)=\left(\prod_{k=0}^{l-2} P\left(h^{k} \mid h^{k+1}\right)\right) P\left(h^{l-1}, h^{l}\right) .
$$

The input data of the DBN model comprise the two-dimensional (2D) vector obtained during preprocessing. The RBM layers were trained one by one in pretraining. The succeeding visible variable was the duplicate of the hidden variable in the previous layer. The parameters transferred in layer-wise manner, and the features were learned from the previous layer. The LR in the highest layer was trained by fine-tuning, where the cost function was revised via back propagation to optimize the weights $w$ [11].

The DBN architecture is shown in Figure 1.

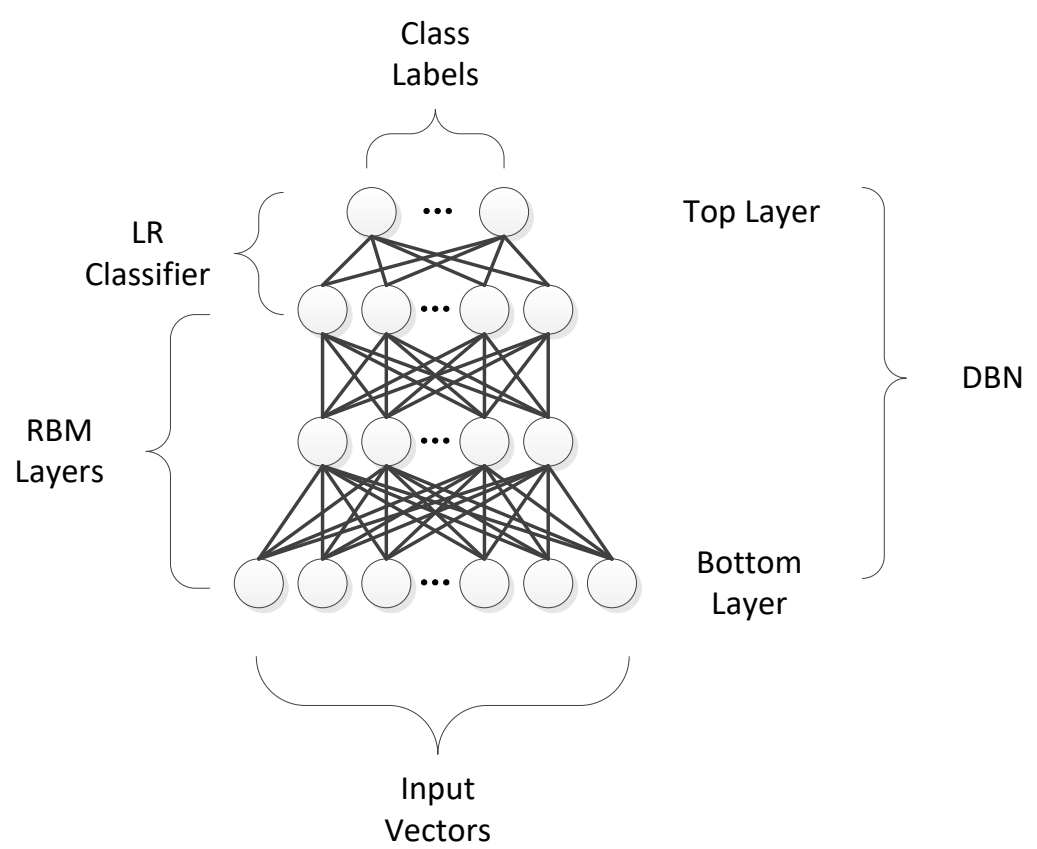

Figure 1. Architecture of a deep belief network (DBN).

Two steps are involved in the process of training a DBN model. Each RBM layer is unsupervisedly trained, the input should be mapped into different feature spaces, and information should be kept as much as possible. Subsequently, the LR layer is added on top of the DBN as a supervised classifier [12].

\subsection{Proposed Method}

Features are the raw materials for training and influence the performance of the final model. Theoretically, the more hidden layers are present, the more features the deep neural network (DNN) can extract, and the more complex the function learned. Accordingly, the DNN model can be described in detail. However, the DNN is gradually replaced by a shallow learning model, such as SVM and boosting, due to problems that occur when the weights $w$ are initialized with a random number in a multi-layer network.

If the weights are set to be too large, then the training process will result in the local optimum. When the weights are set too small, gradient dispersion will occur, and the weights change gradually due to the small gradient. Obtaining the optimal solution is also difficult.

To address these problems, a layer-by-layer initialization of the deep neural network can obtain initial weights that are close to the optimal solution [13]. Layer-by-layer initialization is obtained through unsupervised learning, which can be conducted automatically. 
On the basis of the DBN model, this work proposes a new method of classifying hyperspectral images. The basic DBN classification model involves preprocessing of data, pretraining, and fine-tuning. The difference between DBN and a neural network is the introduction of pretraining. The initial weight value can be close to the global optimization in the DBN model. Therefore, the greedy layer-wise supervised learning has better accuracy than does a neural network. In the supervised fine-tuning procedure, the mini-batch DBN model validates the learned features and the loss function to update the weights $w$. Parameters are trained in mini-batches every training epoch.

Hyperspectral sensor data comprise a spectral image cube that combines spectral and spatial information. Therefore, we compared the effect of two DBN structures, namely SC-DBN and JSSC-DBN. For SC-DBN, input data were the spatial information. Meanwhile, for JSSC-DBN, the input data comprised a new vector that combined the spectral and spatial information [14]. The entire process is presented in Figure 2.

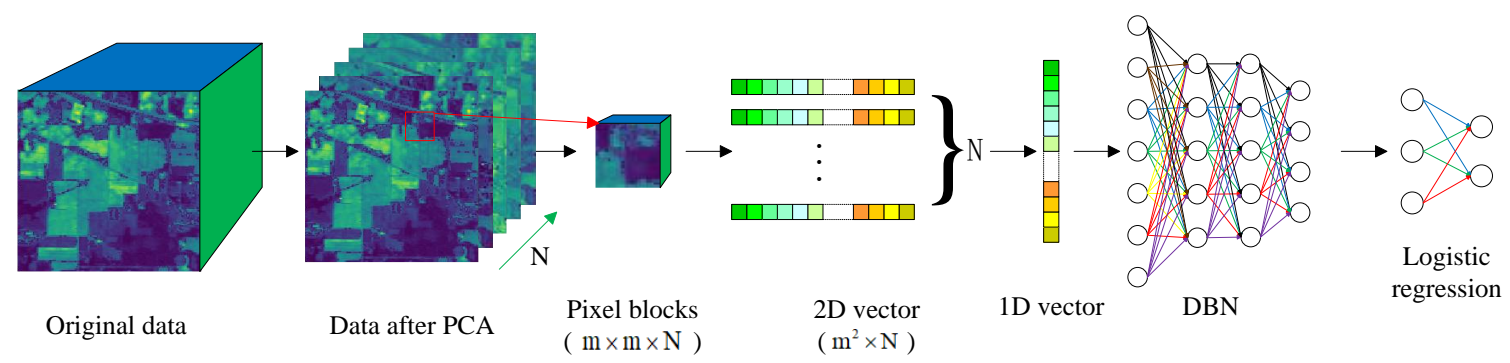

(a) Spatial classifier (SC)-DBN

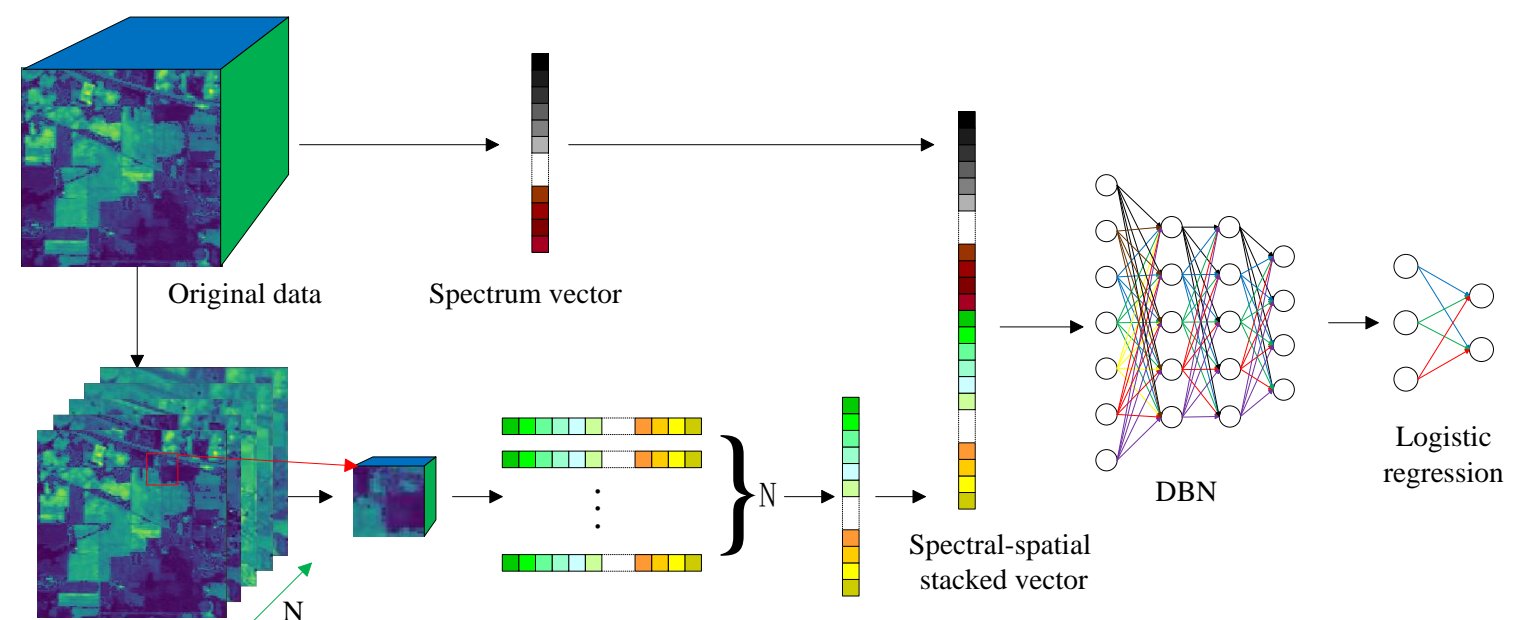

$\begin{array}{lll}\text { Data after PCA } & \begin{array}{l}\text { Pixel blocks } \\ (\mathrm{m} \times \mathrm{m} \times \mathrm{N})\end{array} & \begin{array}{c}2 \mathrm{D} \text { vector } \\ \left(\mathrm{m}^{2} \times \mathrm{N}\right)\end{array}\end{array}$

(b) Joint spectral-spatial classifier (JSSC)-DBN

Figure 2. Process of hyperspectral classification based on DBN.

\subsubsection{Spatial Classification}

Spatial information plays an important role in classification when we attempt to improve accuracy. Before the spatial data are obtained, dimension reduction must be conducted on the hyperspectral remote images. Unlike spectral classification, which extracts spectral information from each pixel, SC cuts an image by window size, as shown in Figure 2a. Firstly, principal component analysis (PCA) dimension reduction was carried out to extract $n$ main components of spectral information. Then, the neighborhood pixel blocks of $m \times m$ were extracted with the labeled pixels as the center, so that each pixel block contained spatial structure information, and the size of the pixel block was $m \times m \times N$. 
After that, the block of pixels was transformed into a 2D feature vector with the size of $m^{2} \times N$. Finally, the 2D feature vector was stretched to a one-dimensional (1D) vector with the size of $m^{2} N \times 1$.

Then, the 1D vector with spatial information was fed into the DBN model. We constructed each RBM layer that shared weighs with each sigmoidal layer. In each layer, CD-k was used in pretraining, so that weights could be updated by errors between the predicted classification results and the true land cover labels. Finally, spatial feature was learned layer by layer. Stochastic gradient descent was used to update the cost function in finetuning. In every epoch, the SC-DBN model calculated the cost on the validation set to make the loss as close as possible to the best validation loss. At the end, we tested it on the test set to obtain accuracy and kappa coefficient.

\subsubsection{Joint Spectral-Spatial Classification}

Joint spectral and spatial classification (JSSC) was long applied in hyperspectral sensor data classification $[15,16]$. In our proposed work, a spectral-spatial classifier was introduced in the deep learning method. To fully use the spectral-spatial information, joint spectral-spatial classification places the spectral and spatial features together in a DBN classifier. Generally speaking, the pixels in the same spatial neighborhood have the same or similar spectral characteristics as the central pixels. Therefore, the neighborhood pixel blocks of $m \times m$ are extracted with the labeled pixels as the center, and each pixel block contains spatial structure information. Then, the pixel blocks are transformed into $2 \mathrm{D}$ feature vectors, and the 2D feature vectors are transformed into $1 \mathrm{D}$ vectors. Meanwhile, the 1D vector containing spectral information is extracted, whose length is equal to the number of spectral bands. The 1D vector with spatial information and the $1 \mathrm{D}$ vector containing spectral information are stitched together into a vector, which is the input of JSSC-DBN.

The spatial information of hyperspectral images has the correlation that the same object will occupy a certain space. Thus, the size of the window should be selected properly. We selected $m \times m$ data and placed an $m \times m \times N$ neighbor region to feed a $m^{2} \times N$ vector into the input layer. We merged spatial data and spectral data into one input vector. Later on, regularization was used in principal component analysis (PCA) when we trained the parameters of the JSSC-DBN model. PCA was conducted to reduce the dimension of the hyperspectral image, while regularization could overcome overfit problem in training. The difference between JSSC and SC is that the former concatenates spatial and spectral vectors to form a new vector, which is then passed to the input layer.

The processing of the multi-layer RBM network in JSSC is discussed above. For the target dataset, the model analysis determined the accuracy of each class and the classification map at the end.

\section{Experiments and Analysis}

\subsection{Dataset and Set-Up}

We used common remote images to verify the effectiveness of the proposed method. One image came from the Pavia University dataset, which was captured by the ROSIS sensor. The ROSIS sensor is a compact, programmable imaging spectrometer based on a CCD (charge-coupied device) matrix detector array. The instrument is specially designed for monitoring water color and natural chlorophyll fluorescence, with the purpose of quantitatively extracting the distribution of pigments, suspended substances, and yellow substances in the marine environment [17]. The scene is sized $610 \times 340$ pixels and has 103 bands (after noisy bands are removed). The geometric resolution is $1.3 \mathrm{~m}$ with nine classes, namely asphalt, bitumen, gravel, sheet, bricks, shadows, meadows, soil, and trees, as shown in Figure 3. The total number of samples, training sets, validation sets, and test sets for each class is detailed in Table 1.

Another example was the Indian Pines dataset, which is sized of $145 \times 145$ pixels and has 224 bands. The image was captured by the AVIRIS sensor. The AVIRIS sensor was flown for the first time in 1986 (first airborne images), and captured first science data in 1987; its data can provide a spatial resolution of $20 \mathrm{~m}$ and 224 spectral bands, covering a spectral range of 0.2-2.4 phenotypes, with 
a spectral resolution of $10 \mathrm{~nm}$ [18]. After de-noising of the original images, the remaining 200 bands were kept without water absorption for the experiments. Figure 4 shows the ground-truth objects. The total number of samples, training sets, validation sets, and test sets for the 16 ground-truth objects of Indian Pines are shown in Table 2.

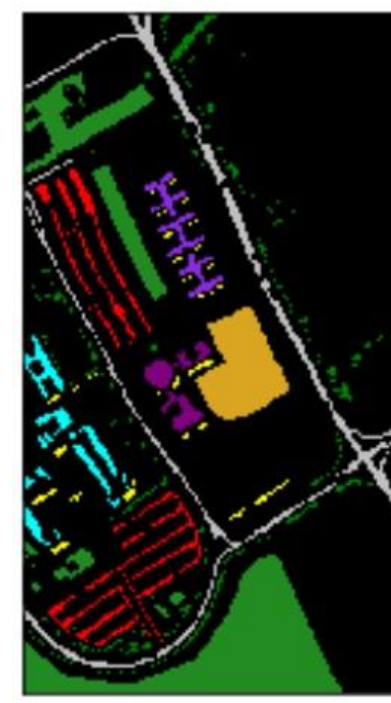

Asphalt

Meadows

Gravel

Trees

Painted metal sheets

Bare Soil

Bitumen

Self-Blocking Bricks

Shadows

Figure 3. Pavia University representing nine classes.

Table 1. Land cover classes and numbers in Pavia University.

\begin{tabular}{cccccc}
\hline$\#$ & Class & Samples & Training & Validation & Test \\
\hline 1 & Asphalt & 6631 & 3979 & 1326 & 1326 \\
2 & Meadows & 18,649 & 11,189 & 3730 & 3730 \\
3 & Gravel & 2099 & 1259 & 420 & 420 \\
4 & Trees & 3064 & 1838 & 613 & 613 \\
5 & Painted metal sheets & 1345 & 807 & 269 & 269 \\
6 & Bare Soil & 5029 & 3017 & 1006 & 1006 \\
7 & Bitumen & 1330 & 798 & 266 & 266 \\
8 & Self-blocking bricks & 3682 & 2210 & 736 & 736 \\
9 & Shadows & 947 & 569 & 189 & 189 \\
\hline Total & & $\mathbf{4 2 , 7 7 6}$ & $\mathbf{2 5 , 6 6 6}$ & $\mathbf{8 5 5 5}$ & $\mathbf{8 5 5 5}$ \\
\hline
\end{tabular}

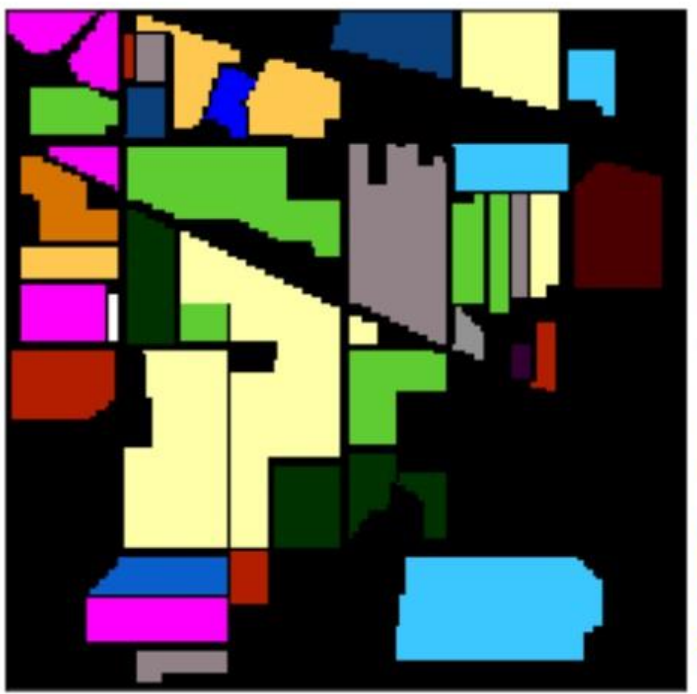

Alfalfa

Corn-notill

Corn-mintill

Corn

Grass-pasture

Grass-trees

Grass-pasture-mowed

Hay-windrowed

Oats

Soybean-notill

Soybean-mintill

Soybean-clean

Wheat

Woods

Buildings-Grass-Trees-Drives

Stone-Steel-Towers

Figure 4. Indian Pines representing 16 classes. 
Table 2. Land cover classes and numbers in Indian Pines.

\begin{tabular}{cccccc}
\hline$\#$ & Class & Samples & Training & Validation & Test \\
\hline 1 & Alfalfa & 46 & 28 & 9 & 9 \\
2 & Corn-notill & 1428 & 856 & 286 & 286 \\
3 & Corn-mintill & 830 & 498 & 166 & 166 \\
4 & Corn & 237 & 143 & 47 & 47 \\
5 & Grass-pasture & 483 & 289 & 97 & 97 \\
6 & Grass-trees & 730 & 438 & 146 & 146 \\
7 & Grass-pasture-mowed & 28 & 16 & 6 & 6 \\
8 & Hay-windrowed & 478 & 286 & 96 & 96 \\
9 & Oats & 20 & 12 & 4 & 4 \\
10 & Soybean-notill & 972 & 584 & 194 & 194 \\
11 & Soybean-mintill & 2455 & 1473 & 491 & 491 \\
12 & Soybean-clean & 593 & 355 & 119 & 119 \\
13 & Wheat & 205 & 123 & 41 & 41 \\
14 & Woods & 1265 & 759 & 253 & 253 \\
15 & Buildings-grass-trees-drives & 386 & 232 & 77 & 77 \\
16 & Stone-steel-towers & 93 & 55 & 19 & 19 \\
\hline Total & & $\mathbf{1 0 , 2 4 9}$ & $\mathbf{6 1 4 7}$ & $\mathbf{2 0 5 1}$ & $\mathbf{2 0 5 1}$ \\
\hline
\end{tabular}

In our experiment, we trained the proposed methods to investigate the influence of the parameters in the DBN network and to improve accuracy. To compare the results of SC and JSSC on the two datasets, we investigated the overall accuracy (OA), average accuracy (AA), and kappa coefficient. The program was run by Python libraries.

The preprocessing step included conducting PCA on the entire data and transforming the spectral and spatial information into a 2D vector. This step rendered the three-dimensional (3D) matrix into the vector, which could be the input of the DBN model.

To avoid potential autocorrelation issues, a validation dataset was added to the training and test data. The ratio of the training, validation, and testing was 6:2:2, as shown in Tables 1 and 2 . The original data were in matrix form but also needed to be normalized. The parameters of the proposed DBN network are shown in Table 3, and the window size of both datasets was $7 \times 7$. The parameters of the SVM were determined using the grid-search algorithm, and we set $c$ to 10,000 and $g$ to 10 [19].

Table 3. The deep belief network (DBN) network parameters.

\begin{tabular}{ccccc}
\hline Dataset & $\begin{array}{c}\text { Number of } \\
\text { Hidden Layers }\end{array}$ & $\begin{array}{c}\text { Number of Hidden } \\
\text { Layer Nodes }\end{array}$ & $\begin{array}{c}\text { Pretrain Learning } \\
\text { Rates }\end{array}$ & $\begin{array}{c}\text { Fine-Tune } \\
\text { Learning Rates }\end{array}$ \\
\hline Indian Pines & 3 & $310 \times 100 \times 100$ & 0.01 & 0.001 \\
\hline Pavia & 3 & $280 \times 100 \times 100$ & 0.05 & 0.003 \\
\hline
\end{tabular}

We performed the experiments 100 times with the original randomized training samples to obtain the experimental results of SVM and the DBN classifier.

\subsection{SC}

In this part of the experiment, we began by classifying the hyperspectral sensor data using the SC-DBN method. We focused on the effect of the number of principals. The number of principal components was selected from 1 to 5 . The pretraining epochs were set to 1000 for Indian Pines and 800 for Pavia University. The result is shown in Figure 5. Evidently, the optimal component was 5 for both datasets. Meanwhile, we investigated the influence of the number of hidden layers, which are also called "depths". Similar experiments were performed on depths not exceeding 5 . 


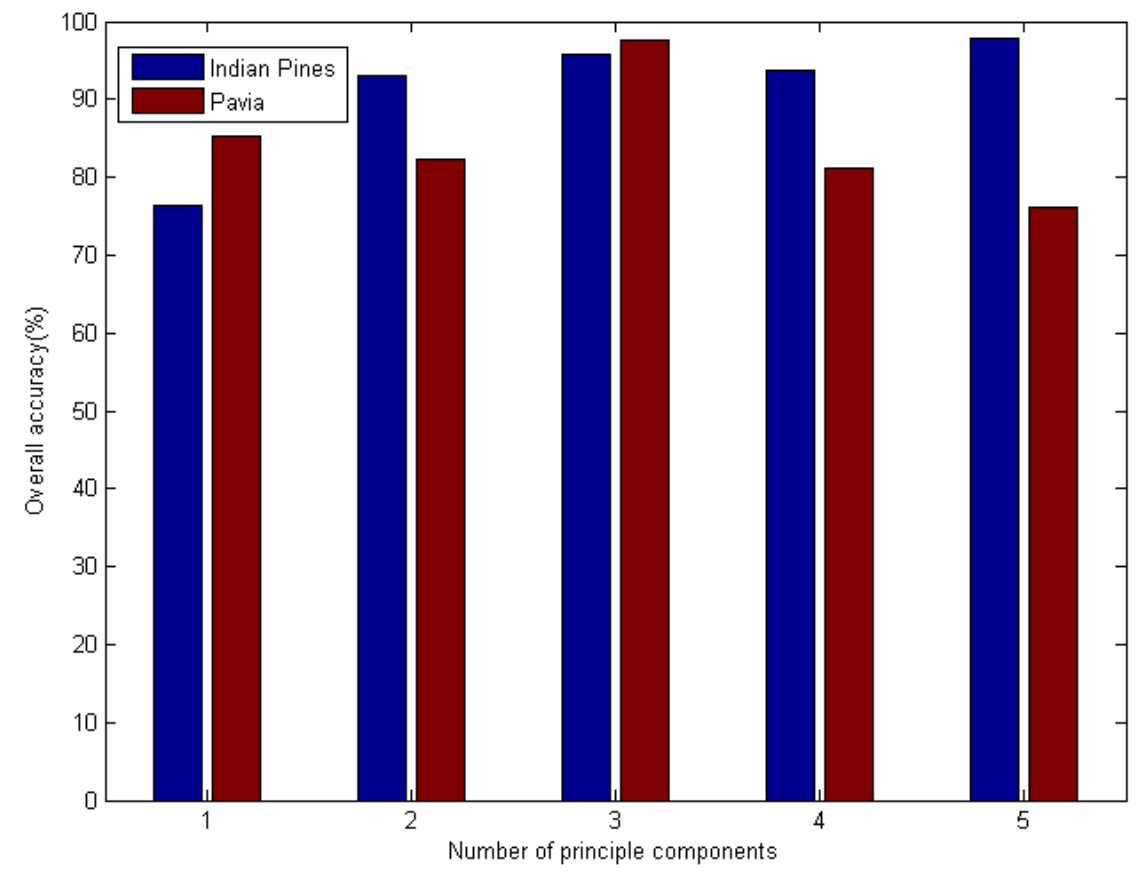

Figure 5. Effect of principal components (SC-DBN classifier).

The detailed classification accuracies and corresponding kappa coefficients of the DBN are shown in Table 4.

Table 4. Overall accuracy (OA), average accuracy (AA), and kappa coefficients of Indian Pines and Pavia University. SC—spatial classifier; JSSC—joint spectral-spatial classifier; SVM—support vector machine.

\begin{tabular}{ccccc}
\hline Dataset & Measurements & SC-DBN $\boldsymbol{n}=\mathbf{3}$ & JSSC-DBN $\boldsymbol{n}=\mathbf{3}$ & SVM \\
\hline \multirow{3}{*}{ Indian Pines } & OA (\%) & 95.81 & 96.29 & 85.71 \\
& AA (\%) & 94.50 & 95.18 & 82.93 \\
& Kappa (\%) & 95.22 & 95.78 & 83.26 \\
\hline \multirow{3}{*}{ Pavia } & OA (\%) & 95.83 & 97.67 & 85.45 \\
& AA (\%) & 94.67 & 96.79 & 80.33 \\
& Kappa (\%) & 94.54 & 96.95 & 80.94 \\
\hline
\end{tabular}

In SC-DBN, feature extraction is difficult. We selected the number of principals in range 5 and overall accuracy was considered as the measurement. According to Figure 5, the number of principal components influenced the accuracy of classification. For Indian Pines, The SC-DBN model performed best when $n$ was 5 . For Pavia University, the best number of principal components was 3 .

The entire image classification result of SC-DBN is shown in Figure 6. From the classification map, different classes are shown in several colors. For SC, the experiments could achieve accuracy of about $97.7 \%$ in Indian and $95.8 \%$ in Pavia. In summary, the classification results indicate that the DBN models performed well on the hyperspectral images. The edge of each class was not notably straight when compared with the ground truth.

\subsection{JSSC}

In this section, we investigated the influence of the number of principal components and hidden layers on spectral-spatial information. Similarly, the number of principal components was researched for JSSC. The testing results of Indian Pines and Pavia datasets are shown in Figure 7. It shows the effect of the number of principal components when using the JSSC-DBN method to classify the 
hyperspectral sensor data. The best numbers of principal components for Indian Pines and Pavia University were 4 and 3, respectively.

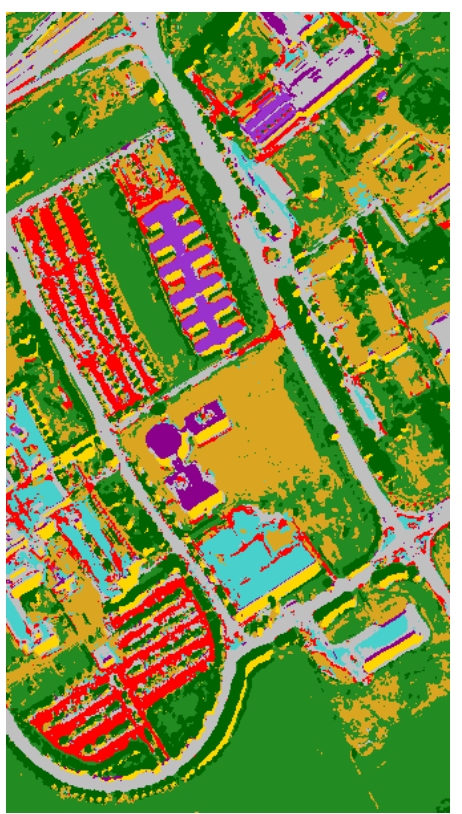

(a)

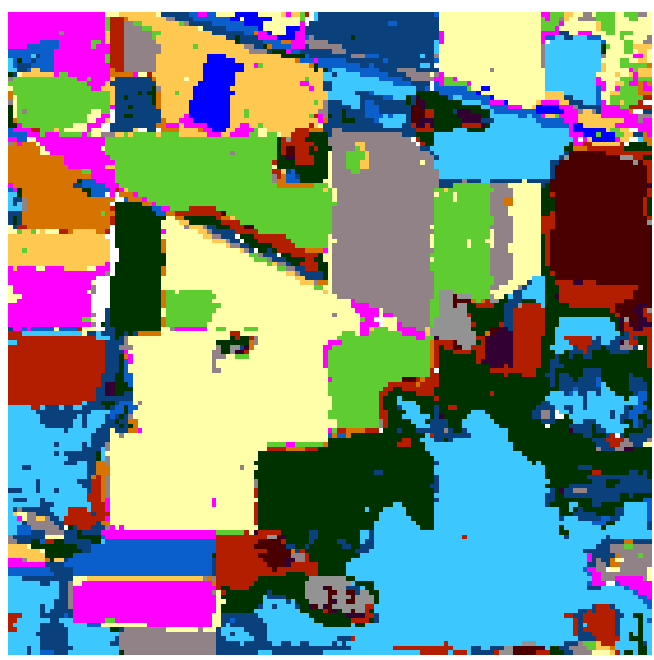

(b)

Figure 6. Spatial information-dominated classification result for Pavia University (a) and Indian Pines (b).

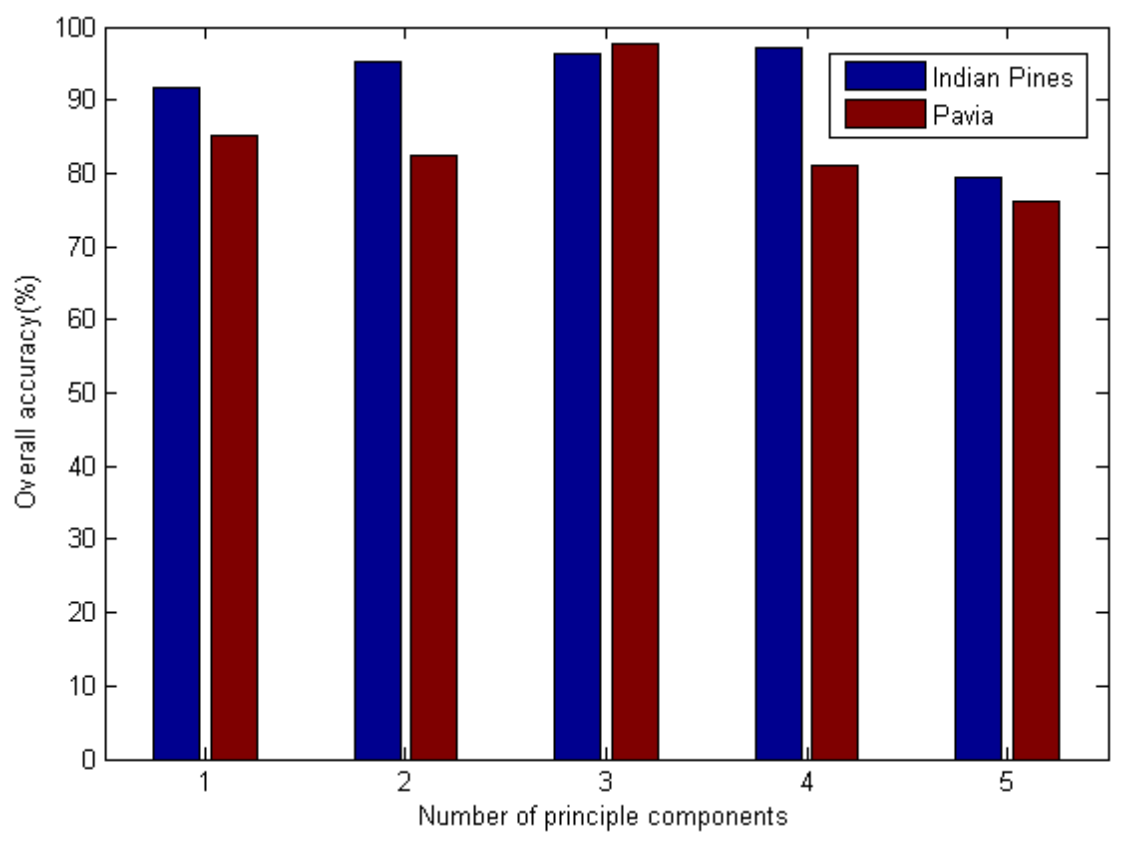

Figure 7. Effect of principal components (JSSC-DBN classifier).

For JSSC, the experiments could achieve overall accuracy of about $97.7 \%$ in Indian and $95.8 \%$ in Pavia. Therefore, the DBN model is a promising method for classifying hyperspectral images, whether using SC or JSSC.

The joint-dominated classification maps on Pavia University and Indian Pines are shown in Figure 8 . 


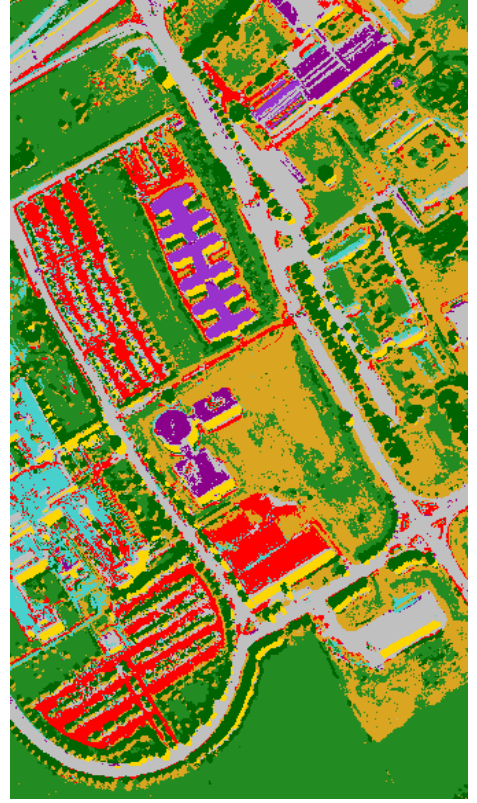

(a)

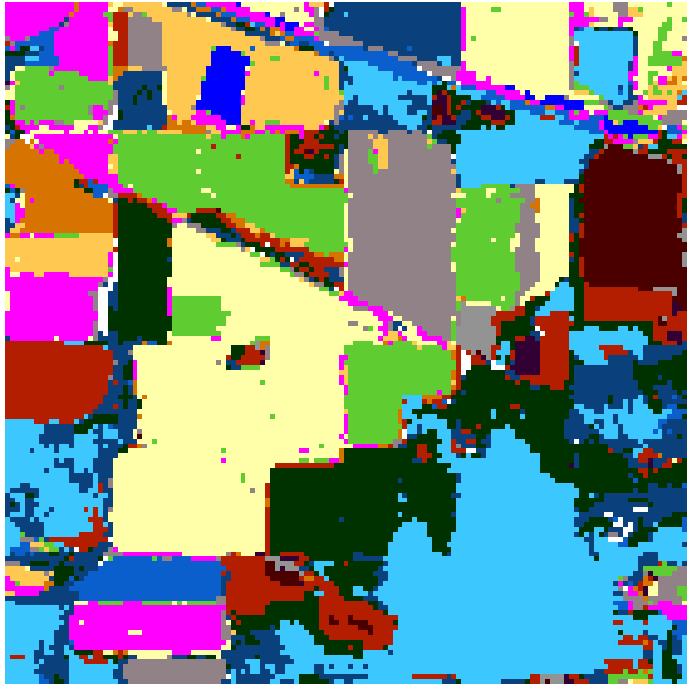

(b)

Figure 8. Joint-dominated classification result for Pavia University (a) and Indian Pines (b).

In terms of the SVM-based method, spatial and spectral classification could also obtain high accuracy. The origin data were split into two parts for the verification of the classification results and calculation of the AA, thus comparing the SVM-based method and the proposed technique.

Detailed classification accuracies and corresponding kappa coefficients of each class are shown in Table 5. As the JSSC can extract more information than SC and SVM, it is unsurprising to find that JSSC performed the best among the three classifiers.

Table 5. Classification result on Indian Pines.

\begin{tabular}{cccc}
\hline Class & SC-DBN $(n=4)$ & JSSC-DBN $(n=4)$ & SVM \\
\hline Alfalfa & 100 & 100 & 33.33 \\
Corn-notill & 92.71 & 96.87 & 94.44 \\
Corn-mintill & 86.93 & 94.01 & 77.84 \\
Corn & 95.56 & 100 & 88.89 \\
Grass-pasture & 97.03 & 100 & 91.09 \\
Grass-trees & 96.53 & 98.26 & 91.33 \\
Grass-pasture-mowed & 85.71 & 100 & 28.57 \\
Hay-windrowed & 100 & 100 & 97.94 \\
Oats & 100 & 100 & 33.33 \\
Soybean-notill & 86.01 & 98.93 & 84.46 \\
Soybean-mintill & 97.59 & 97.73 & 100 \\
Soybean-clean & 85.59 & 92.03 & 66.95 \\
Wheat & 95.83 & 97.91 & 91.67 \\
Woods & 99.21 & 98.41 & 91.67 \\
Buildings-grass-trees-drives & 80.25 & 89.23 & 27.16 \\
Stone-steel-towers & 100 & 100 & 0.00 \\
Kappa (\%) & 92.82 & 96.88 & 83.26 \\
Overall accuracy (\%) & 93.71 & 97.26 & 85.71 \\
Average accuracy (\%) & 93.68 & 96.28 & 83.08 \\
\hline
\end{tabular}

\section{Conclusions and Discussion}

In this work, we proposed a new hyperspectral image classification model based on DBN. The proposed model learns deep features during hyperspectral image classification. The framework of 
a DBN was introduced to classify spatial hyperspectral sensor image data on the basis of the DBN. Then, the improved method, which combines spectral and spatial information, was verified. After unsupervised pretraining and supervised fine-tuning, the DBN model could successfully learn the features. We added an LR layer on top for classifying the hyperspectral images. In comparison with the SVM-based method, the DBN model performed better in terms of accuracy and kappa coefficient. In addition, JSSC-DBN was proven to be the best classifier.

Therefore, the deep learning method improves the accuracy of hyperspectral classification. According to our results, we suggest that the DBN model be designed with 3-5 hidden layers, with each having no more than 100 hidden units.

In our future work, we will improve the DBN model regarding its accuracy and time consumption. Given the irreplaceable role of spectral-spatial feature extraction in the DBN model, further investigation should be devoted to parameter optimization of the deep learning framework. The DBN model runs slowly; thus, the PCA algorithm was selected to reduce the dimension of hyperspectral data, before being input into the DBN model designed in this paper for classification. However, the performance of the PCA algorithm in classification tasks is not ideal. In the future, we will continue improving the model in combination with the latest achievements in the field of dimensionality reduction algorithms.

Author Contributions: C.L. and H.G. conceived and designed the experiments; X.Z. and Y.Y. presented tools and carried out the data analysis; H.G. and Y.W. wrote the paper. C.L. and J.W. guided and revised the paper.

Funding: This research was funded by the National Natural Science Foundation of China (No. 61701166, No. 41601435), the China Postdoctoral Science Foundation (No. 2018M632215), the Young Elite Scientists Sponsorship Program by CAST (No. 2017QNRC001), the National Science Foundation for Young Scientists of China (No. 51709271), Science Fund for Distinguished Young Scholars of Jiangxi Province under Grant (No.2018ACB21029), and the Projects in the National Science \& Technology Pillar Program during the Twelfth Five-Year Plan Period (No. 2015BAB07B03).

Conflicts of Interest: The authors declare no conflicts of interest.

\section{References}

1. Li, H.; Xiao, G.; Xia, T.; Tang, Y.Y.; Li, L. Hyperspectral image classification using functional data analysis. IEEE Trans. Cybern. 2014, 44, 1544-1555. [PubMed]

2. Bazi, Y.; Melgani, F. Toward an optimal SVM classification system for hyperspectral remote sensing images. IEEE Trans. Geosci. Remote Sens. 2006, 44, 3374-3385. [CrossRef]

3. Zhao, W.; Guo, Z.; Yue, J.; Zhang, X.; Luo, L. On combining multiscale deep learning features for the classification of hyperspectral remote sensing imagery. Int. J. Remote Sens. 2015, 36, 3368-3379. [CrossRef]

4. Hinton, G.E.; Osindero, S.; Teh, Y.W. A fast learning algorithm for deep belief nets. Neural Comput. 2006, 18, 1527. [CrossRef] [PubMed]

5. Goudarzi, S.; Kama, M.N.; Anisi, M.H.; Soleymani, S.A.; Doctor, F. Self-organizing traffic flow prediction with an optimized deep belief network for internet of vehicles. Sensors 2018, 18, 3459. [CrossRef] [PubMed]

6. Norouzi, M.; Mani, R.; Greg, M. Stacks of convolutional restricted Boltzmann machines for shift-invariant feature learning. In Proceedings of the 2009 IEEE Conference on Computer Vision and Pattern Recognition, Miami, FL, USA, $20-25$ June 2009; Volumes 1-4, pp. 2727-2734.

7. Sun, C.; Yang, Y.; Wen, C.; Xie, K.; Wen, F. Voiceprint identification for limited dataset using the deep migration hybrid model based on transfer learning. Sensors 2018, 18, 2399. [CrossRef] [PubMed]

8. Cho, K.H.; Raiko, T.; Ilin, A. Gaussian-Bernoulli deep boltzmann machine. In Proceedings of the 2013 International Joint Conference on Neural Networks (IJCNN 2013), Dallas, TX, USA, 4-9 August 2013; pp. 1-7.

9. Hinton, G.E.; Roweis, S. Modeling human motion using binary latent variables. In Proceedings of the 2006 Conference on Advances in Neural Information Processing Systems 19, Vancouver, BC, Canada, 4-7 December 2007; pp. 1345-1352.

10. Hinton, G.E. Training products of experts by minimizing contrastive divergence. Neural Comput. 2002, 14, 1771. [CrossRef] [PubMed] 
11. Hecht-Nielsen, R. Theory of the backpropagation neural network. In Proceedings of the International Joint Conference on Neural Networks (IEEE, 2002), Honolulu, HI, USA, 12-17 May 2002; Volume I, pp. 593-605.

12. Schölkopf, B.; Platt, J.; Hofmann, T. Greedy Layer-Wise Training of Deep Networks. International Conference on Neural Information Processing Systems; MIT Press: Cambridge, MA, USA, 2006; pp. 153-160.

13. Bengio, Y.; Delalleau, O. On the expressive power of deep architectures. In Proceedings of the 22nd International Conference on Algorithmic Learning Theory (ALT 2011), Espoo, Finland, 5-7 October 2011.

14. Pu, H.; Chen, Z.; Wang, B.; Geng-Ming, J. A novel spatial-spectral similarity measure for dimensionality reduction and classification of hyperspectral imagery. IEEE Trans. Geosci. Remote Sens. 2014, 52, 7008-7022.

15. Zhao, W.; Du, S. Spectral-spatial feature extraction for hyperspectral image classification: A dimension reduction and deep learning approach. IEEE Trans. Geosci. Remote Sens. 2016, 54, 4544-4554. [CrossRef]

16. Fauvel, M.; Tarabalka, Y.; Benediktsson, J.A.; Chanussot, J.; Tilton, J.C. Advances in spectral-spatial classification of hyperspectral images. Proc. IEEE 2013, 101, 652-675. [CrossRef]

17. Doerffer, R.; Kunkel, B.; van der Piepen, H. ROSIS-An imaging spectrometer for remote sensing of chlorophyll fluorescence. Proc. SPIE Int. Soc. Opt. Eng. 1989, 1070. [CrossRef]

18. Li, C.; Yang, S.X.; Yang, Y.; Gao, H.; Zhao, J.; Qu, X.; Wang, Y.; Yao, D.; Gao, J. Hyperspectral remote sensing image classification based on maximum overlap pooling convolutional neural network. Sensors 2018, 18, 3587. [CrossRef] [PubMed]

19. Fauvel, M.; Chanussot, J.; Benediktsson, J.A.; Sveinsson, J.R. Spectral and spatial classification of hyperspectral data using SVMs and morphological profiles. In Proceedings of the 2007 Geoscience and Remote Sensing Symposium (IGARSS 2007), Barcelona, Spain, 23-27 July 2007; pp. 4834-4837.

(C) 2019 by the authors. Licensee MDPI, Basel, Switzerland. This article is an open access article distributed under the terms and conditions of the Creative Commons Attribution (CC BY) license (http:// creativecommons.org/licenses/by/4.0/). 